\title{
Primary Exploration on Speed and Endurance Training for Men's 400m Athletes
}

\author{
Yiming Zou
}

Aviation Security Institute, Civil Aviation Flight University of China, Guanghan, 618307, China

Keywords: Men's 400m. Athletes. Speed. Endurance. Training

\begin{abstract}
In view of rapid development of global track and field sports, more and more attention is paid to competition result. Although Chinese level of track and field sports is slightly improved, the level of men's 400m race is still not high. In order to improve sport performance and reach the global leading level, it is very necessary to implement speed and endurance training in a scientific and systematic way. This paper analyzes the development prospect of modern sprint technology, introduces the basic requirements for speed and endurance training of men's 400m race, analyzes the factors influencing men's 400m athletes' speed and endurance, discusses the training method of improving men's 400m athletes' speed and endurance, and proposes the problems which shall be noticed in the speed and endurance training for men's 400m athletes.
\end{abstract}

\section{Introduction}

The men's 400m race is a short-distance track and field sport event; the speed and endurance are very important content in the training of such event, and also the necessary condition to improve the performance. In terms of sports biochemistry performance of men's $400 \mathrm{~m}$ race, the energy supply is determined by the content of adenosine triphosphate, phosphocreatine, and sugar, etc. Therefore, in the training for men's $400 \mathrm{~m}$ athletes, the full attention shall be paid to training on athletes' anaerobic ability and lactic acid energy ability, etc. so as to strive for best training effect.

\section{Analysis on development prospect of modern sprint technology}

Firstly, more attention is paid to standardized actions of sprint technology. The main performance is that the sprint technology more conforms to the principles of sports biomechanics and sports anatomy, which makes the sprint technology show stronger efficiency and rationality. The structural style of sprint technology is mainly reflected on high stride frequency and large stride width; the action is natural and steady, the center of gravity has small up and down motion, and the upper and lower limbs have harmonious coordination in terms of action and show obvious sense of rhythm. Secondly, more attention is paid to swing technique of sprint technology. Such technique includes arm swing and leg swing technique. In terms of new sprint technology, we shall pay attention to the important significance that the swing technique has. In training structure, we shall enhance the exploration on swing actions, so as to get hold of rules and biomechanical features that the swing technique has, and accurately understand the biomechanical theory that the arm and leg swing technique has. Thirdly, the speed improvement shall start from athletes' hip. American experts Sprague and Wood et al. obtained the opinions according to mechanics principle: stretching muscles of hip plays an very important role in speed. Moreover, Ayer and other scholars applied high-speed photography, explanation on sport technique and other modern science and technology methods to carry out contrastive analysis on speed of movement of lower limbs between the world first-class sprinter and common sprint amateur, and found that the main difference was that the world high-level athletes had a quicker hip muscle stretching speed than common sprint amateur. This research result provides a powerful evidence for developing power to stretch muscles of hip in the training for sprint athletes. 


\section{Basic requirements for speed and endurance training of men's $400 \mathrm{~m}$ race}

The speed training shall be carried out according to the sport event that the athletes engage in. As for speed training of $400 \mathrm{~m}$ race, the attention shall be paid to improving athletes' auditory reaction force. The speed training shall be carried out under the condition that the sprint athletes are under a stage of excitation and have high emotion and sport desire, and it is mainly arranged at the first half part of training. When the speed is improved to one stage, there will be no improvement, which is also called speed barrier. In the training process, it is required to carefully analyze subjective and objective reason which results in speed barrier, and the effective method shall be adopted to practically solve it.

In the endurance training, we shall pay attention to following two points. Firstly, we shall actively cultivate athletes' breathing ability. In the process of endurance training, we shall pay special attention to breathing problem; the athletes make use of breathing to take sufficient oxygen which can maintain the work for a relatively long time, and the human body obtains more oxygen mainly through improving breathing frequency and deepening breathing concentration. Under general condition, in the process of long-time work, the high-level sprint athletes improve oxygen supply mainly through improving breathing depth and reducing breathing times, etc. In the process of implementing endurance training, it is required to enhance development of athletes' habit of breathing through nose. From perspective of sanitation, the mucosa of nasal cavity can purify the inhalational air, so as to prevent dust and cold air from directly entering into lung. Secondly, the development of volitional quality shall be enhanced. The sprint athletes' volitional quality plays a very important role in the process of endurance training; in particular, when the training for athletes is up to ultimate strength, whether the athletes can insist on training will depend on their volitional quality. Therefore, in the training of endurance quality, it is required to enhance the improvement of sprint athletes' volitional quality, especially the environment with high temperature or low atmospheric pressure will cause great influence on people's endurance. In order to resist against those adverse factors, it is very necessary for athletes to own powerful volitional quality.

\section{Factors influencing men's 400m athletes' speed and endurance}

The Russian scholar Sultanov proved through research that $10 \%$ of energy supply depended on aerobic working ability, and the remaining energy supply depended on anaerobic working ability in the energy supply of $400 \mathrm{~m}$ race. The improvement of speed mainly depends on improvement of lactic acid energy; at the starting stage of people's muscular movement, all energies are supplied through decomposition of ATP and CP. The energy supply through decomposition of ATP doesn't need involvement of oxygen and doesn't generate water. If large part of energy supply is from decomposition of ATP, CP plays a supplementary role, and it promotes ATP to make re-synthesis. However, there are only few content of ATP and CP, which can only maintain ultimate amount of exercise for $10 \mathrm{~s}$ at most; this is equal to a distance of $80 \mathrm{~m}-90 \mathrm{~m}$ under condition of sprint. Besides, the energy supply time of ATP and CP is not more than 30s at most, which can't meet the whole journey demand of $400 \mathrm{~m}$ race, thus another energy system and lactic acid energy are required to supply energy. Once the large-strength sport is continued for 6-8s, the glycolysis will be activated; if the time is more than 10s, the proportion of glycolysis will increase, and the muscle glycogen will be quickly decomposed into water and participate in energy supply in sport process, that is to say, the lactate dehydrogenase shall be conversed into pyruvic acid through effective application of blood so as to realize oxidation and energy supply; the energy supply time can be maintained for 2-3 minutes at most so as to meet the whole-journey demand of $400 \mathrm{~m}$ race. Therefore, the $400 \mathrm{~m}$ race shall be carried out through the energy supplied by two systems, ATP-CP system and lactic acid energy. Under general condition, the main reason why the $400 \mathrm{~m}$ race event can't be maintained for a long time is that the glycolysis is generated into lactic acid while it is generated into ATP; once the lactic acid accumulates and quickly increases in human body, some changes will happen in internal environment, which restrains the activity of glycolytic enzyme. When $\mathrm{PH}$ value is $6.4 \sim 6.5$, the activity of main 
oxidase and phosphofructokinase in glycolysis almost completely loses, and ATP generation will be restrained accordingly, which results in continuous decrease of athletes' ability. Therefore, it is very important to improve men's 400m athletes' no-oxygen oxidizability; the ability is mainly reflected at continuous increase of people's content of muscle glycogen when people are quiet, continuously strong activity of lactate dehydrogenase, and increase in blood sugar content in the movement process. Although men's $400 \mathrm{~m}$ race belongs to anaerobic metabolism event, in order to improve the performance of this project, it is required to take oxygen metabolism as premise, and give a comprehensive consideration on complete energy system so as to improve level.

\section{Training method of improving men's 400m athletes' speed and endurance}

The speed training for men's 400m athletes can be good for improving the flexibility of athletes' nerve and muscular activity, and such training plays a decisive role in improving the performance of $400 \mathrm{~m}$ race. Therefore, the speed training is listed as the primary task of $400 \mathrm{~m}$ race training. The speed of $400 \mathrm{~m}$ race is mainly divided into starting response speed, movement speed, and displacement speed. As for training of response speed and movement speed, it is required to carry out special exercise of back-and-forth contact-to-earth running, carry out various kinds of responses and exercises of game property, adopt the quickest speed to develop arm swinging exercise, and carry out high-frequent high-knee training, and the continuous time shall be up to 8-10s. As for training of displacement speed, the repetition method shall be used, that is to say, the athletes take a training of repeated constant distance for many times at a certain speed, and the intensity of exercise is the main factor to improve people's quick movement ability. The strength of displacement is a kind of ultimate strength, and it is generally controlled at $90 \%-95 \%$. In the process of training, before next exercise, the athletes shall take basic recovery of their body as main standard.

The endurance training method for men's 400m athletes shall follow the principle of exercise physiology, that is, the amount of oxygen required in the exercise process depends on strength of movement. The larger the strength is, the larger the amount of oxygen required is; therefore, there will be a larger proportion of anaerobic glycolysis in energy supply. Therefore, the following methods can be applied in the endurance training of men's $400 \mathrm{~m}$ race. Firstly, the running strength is applied to develop the anaerobic ability of glycolysis. It is able to make use of repeated running within $100 \mathrm{~m}-150 \mathrm{~m}$ to carry out training; the strength shall be close to the athlete's quickest running speed, and the interim time shall be about 1 minute. At the beginning, there shall be not many running times, and 10-15 times are suitable. As the sprint athletes have fully adapted to load, on the basis that there is no obvious increase in their blood lactic acid, it is required to increase the repetition times or shorten interim time. Secondly, it is required to adopt short-distance running or long-distance running to develop the athletes' anaerobic ability of glycolysis. There is difference in the time that the glycolysis maintains quick movement of human body's muscles, and the time is generally 40s or 90s. Therefore, when the athletes select the running distance, there shall be also some difference. As for the former, $200 \mathrm{~m}$ with $90 \%-95 \%$ of maximum strength is adopted to carry out exercise of repeated running, and there shall be 8-12 repetition times, with an interim time of about 150s. As for the latter, $600 \mathrm{~m}$ with $85 \%-90 \%$ of strength shall be adopted to carry out exercise, and there shall be $3-4$ repetition time, with an interim time of 4-5 minutes.

\section{Problems which shall be noticed in speed and endurance training for men's $400 \mathrm{~m}$ athletes}

Firstly, the attention shall be paid to improvement of athletes' anaerobic ability and speed. The anaerobic and aerobic change process is mutually related and dependent. In the men's $400 \mathrm{~m}$ race, the anaerobic energy supply is up to $80 \%$; therefore, on the basis of implementing aerobic exercise, more attention shall be paid to anaerobic training. As for men's $400 \mathrm{~m}$ athletes, the training shall be implemented according to middle/long-distance training way; although athletes' sport performance can be improved, the effect is not obvious. This is because the men's $400 \mathrm{~m}$ race is a physical fitness track and field event which pays key attention to speed, and the athletes shall have quick running ability, best speed and endurance, and muscle strength. Therefore, in the training for men's 400m 
athletes, we shall not only pay attention to develop their endurance, but also pay attention to the improvement of absolute speed under the condition of anaerobic ability.

Secondly, the attention shall be paid to training of special strength quality. To have good physical quality has become the important precondition for contemporary athletes to get hold of modern sports skills. The key point of training for $400 \mathrm{~m}$ race athletes is to develop the power of front muscle combination and back muscle combination of thigh, the power of waist, and the power of muscle combination of upper arms, etc.; the required power includes explosive power, quick endurance power, ability of alternation between quick contraction and relaxation of muscle. In the training, the special exercises such as long-distance jumping and running can be implemented. For example, the athletes can make a leap step of 100-200m, skip step of 400-600m, and high-knee step of 500m, etc. Certainly, we shall also pay attention to training of waist and abdominal muscle power; otherwise it will be difficult for athletes to adapt to the intensive competition, which results in transformation of action and then affects good exertion of technique.

Thirdly, the attention shall be paid to regulation and control of training rhythm. The high-strength training can make all organs and systems of human body form adaptive change, so as to realize more active training effect. However, in case of excessive training load, 3 days later, the athletes' sport performance will gradually decreases; after more than 6 days, the athletes can recover their performance to original level. Therefore, the coach shall actively organize the training process, and pay attention to proper arrangement of training rhythm. In each training, the middle and small exercise load shall be suitable used for regulating the training, so as to make their cardiovascular system realize good recovery and avoid occurrence of excessive training.

Fourthly, the comprehensive preparation activity shall be made before training. The preparation activity can pre-motivate people's physiological function, promote muscular extensibility and elasticity, and practically reduce viscosity of muscle, thus shortening the adapting time and exerting the best work efficiency in the training to carry out function preparation. Except for improving the work efficiency of muscle, the preparation activity is also the main means to prevent athletic injury. It is suitable to set the strength as $45 \%$ of maximal oxygen uptake; the situation that the whole body becomes warm and slightly sweats is used as main standard.

\section{Conclusion}

In conclusion, more attention shall be paid to the influence of technical factors on speed and endurance of men's 400m race; this is because good sport technique can conform to human body's morphological anatomy feature and principle of biomechanics, and realize saving of energy so as to obtain ideal mechanics effect, thus the athletes can run a quicker speed. Meanwhile, through continuously perfecting the technique and saving the energy, it is able to enhance reserve of energy, so as to produce effective help for maintaining the ability of high-speed movement. It can be seen that it is required to continue to improve the running technique, and properly handle the proportion relation between speed and endurance well in the process of speed and endurance training of $400 \mathrm{~m}$ race.

\section{References}

[1] Sun Yi: Analysis on Men’s 400m Speed and Endurance Training Method, Modern Science, 2009 (18).

[2] Zhang Kai: Experimental Study on Speed and Endurance Training for Teenager's 400m Athletes, Heilongjiang Science and Technology Information, 2010 (32).

[3]. Li Changdong: Exploration and Analysis on Key Section of 400m Speed and Endurance Training, Sichuan Sports Science, 2011 (2).

[4] Xu Licun: Discussion on Men's 400m Speed and Endurance Quality Training Methods and Means, Science \& Technology Vision, 2012 (8).

[5] Zhong Shaoting: Speed and Endurance Breakthrough Training Methods for Excellent 400m Athletes, Science and Technology in Modern Physical Education, 2012 (9). 